\title{
Effect of incubation temperature on eggs and larvae of lumpfish (Cyclopterus lumpus)
}

\section{Albert Kjartan Dagbjartarson Imsland ${ }^{1,2 *}$, Mathias Danielsen ${ }^{3 \S}$, Thor Magne Jonassen ${ }^{4}$, Thor Arne Hangstad ${ }^{4}$, Inger-Britt Falk- Petersen $^{3}$}

\author{
${ }^{1}$ Akvaplan-niva Iceland Office, Akralind 4, 201 Kópavogur, Iceland \\ ${ }^{2}$ Department of Biology, University of Bergen, High Technology Centre, 5020 Bergen, Norway \\ ${ }^{3}$ Department of Arctic and Marine Biology, University of Tromsø, 9019 Tromsø, Norway \\ ${ }^{4}$ Akvaplan-niva, Framsenteret, 9296 Tromsø, Norway
}

Running title: The effect of incubation temperature on early development in lumpfish

Keywords: Lumpfish; Temperature regime; Hatching success; Larval quality

* Corresponding author at: Akvaplan-niva Iceland Office, Akralind 4, 201 Kópavogur, Iceland. E-mail address: aki@akvaplan.niva.no (A.K. Imsland).

${ }^{\S}$ Equal authorship between: Imsland and Danielsen 


\section{Abstract}

2 Two batches of lumpfish eggs were incubated at three temperature regimes; 1-Ambient 3 seawater $4-6^{\circ} \mathrm{C}$ (cold), 2-Ambient seawater for 10 days followed by a gradual increase to $10^{\circ} \mathrm{C}$

4 (gradient), 3-Constant $10^{\circ} \mathrm{C}$ seawater (warm). The eggs incubated in cold water had the 5 highest egg mortality $(38.5 \% \pm 15.7)$ and lowest hatching success $(46.1 \% \pm 7.2)$, while the 6 gradient group regime showed highest hatching success $(74.9 \% \pm 4.2)$. Larvae from the 7 gradient regime showed the most synchronized hatching as hatching started at $280 \mathrm{dd}$ (35 days 8 post fertilization (DPF)) and reached the hatching peak the same day with almost $80 \%$ of all

9 larvae hatching. Hatching started at $279 \mathrm{dd}(28 \mathrm{DPF})$ in the warm regime, reached a hatching 10 peak (50\% of total hatching) at 3 days post hatch (DPH), and ended at 9 DPH. In the cold 11 temperature group hatcing started at $285 \mathrm{dd}(63 \mathrm{DPF})$ and the hatching peak was reached at 3 12 DPH. Hatching lasted until 13 DPH. Hatched larvae from the cold regime were longest (6.11 $13 \mathrm{~mm})$ and heaviest $(5.55 \mathrm{mg})$, followed by larvae from the gradient $(5.71 \mathrm{~mm}, 4.88 \mathrm{mg})$ and 14 warm $(5.33 \mathrm{~mm}, 4.37 \mathrm{mg})$ regimes respectively. Newly hatched larvae from the warm group 15 had the highest occurrence $(34.7 \%)$ of body deformities compared to 8.9 and $7.6 \%$ in the 16 gradient and cold water groups. Studies of organ and tissue histomorphology of hatched and 17 two weeks old larvae did not reveal obvious developmental differences between the groups at 18 these timepoints. 


\section{1. Introduction}

3 Cleaner fish, like wrasses (Labridae) and lumpfish (Cyclopterus lumpus L.), may

4 represent sustainable solutions for reducing the lice problem in the salmon industry (Treasurer,

5 2002; Imsland et al., 2014a-c, 2015a-b). Due to the wrasses' southern distribution and the fact

6 that their appetite is reduced at low temperature, use of wrasses in the northern parts of Norway

7 is a challenge (Durif, 2015). The lumpfish has a more widespread natural distribution, and is

8 found further north than the northernmost species of wrasses (Davenport, 1985; Durif, 2015).

9 Consequently, the common lumpfish has been suggested as a cold-water cleaner-fish, and

10 initial results are very promising with up to $93-97 \%$ less lice infection (adult female lice) in 11 sea-pens with lumpfish (Imsland et al. 2014a-c; 2015a-b).

12 In nature, from February and onwards in the spring, sexually mature lumpfish return

13 from open waters to spawn at shallow localities in coastal areas (Davenport, 1985; Moen and

14 Svensen, 2004; Durif, 2015). Females spawn in several batches and have relatively high

15 fecundity, laying between 100.000-400.000 demersal eggs in total (Brown, 1992; Moen and

16 Svensen, 2004). Males guard the egg clutches, each of which can be from several females.

17 The eggs are 1.8-2.6 mm in diameter and can have a variety of colours; pink, orange, yellow,

18 green, brown and red and stick to each other after exposure to saltwater (Davenport, 1985;

19 Davenport and Thorsteinsson, 1989; Moen and Svensen, 2004). The larvae hatch after 40 days

20 at $5^{\circ} \mathrm{C}$ (200 day-degrees (dd), Davenport, 1983) and 25 days at $9.8^{\circ} \mathrm{C}(245 \mathrm{dd}$, Collins, 1978).

21 Recent data from the lumpfish industry have found egg development time to be nearer 290 to $22300 \mathrm{dd}$.

23 Several studies show that temperature as a physiological factor has an effect on 24 development and survival of fish eggs and larvae (see e.g. Hansen and Falk-Petersen, 2001; 
1 Sund and Falk-Petersen, 2005; Geffen et al., 2006; Jonsson and Jonsson, 2014). Fish from

2 temperate zones appear to be more sensitive to temperature changes during early life than

3 juveniles and adults (Rombough, 1997). Furthermore, marine fish embryos are suggested to

4 have four periods particularly sensitive to temperature during development: cleavage, early

5 gastrula, embryo appearance and blastopore closure (Kazuyuki et al., 1988). Few scientific

6 studies exist on temperature tolerances of early life stages of lumpfish. Collins (1978) found

7 that lumpfish eggs incubated at average temperatures of $6.4^{\circ} \mathrm{C}$ and $9.8^{\circ} \mathrm{C}$ hatched after 31 and

825 days respectively, and eggs incubated at an average temperature of $3.8^{\circ} \mathrm{C}$ did not hatch at

9 all. Initial production protocols at one of the pioneer production facilities of lumpfish juveniles

10 in northern Norway (Research and Innovation Centre Kraknes, Troms $\varnothing$ ), included egg

11 incubation at both ca. $4^{\circ} \mathrm{C}$ and $10^{\circ} \mathrm{C}$. Both temperatures resulted in relatively high hatching

12 percentages, but larvae from eggs incubated at $4^{\circ} \mathrm{C}$ hatched less synchronously and the eggs

13 were also suffered from higher loads of microorganisms on the surfaces (Thor Arne Hangstad,

14 Research and Innovation Centre Kraknes, pers. comm.). Studies of effects of incubation

15 temperature are important in order to optimize rearing conditions for successful cultivation of

16 good quality juveniles.

17 The objective of this study was to investigate how different incubation temperature

18 regimes influenced egg development, mortality, hatching success and early larvae size,

19 deformities and histomorphology of lumpfish larvae. 


\section{2. Materials and Methods}

\section{$3 \quad 2.2$ Experimental fish and set-up}

4 Sexually mature wild lumpfish were caught by gill nets in Sandnessundet outside

5 Kraknes, Troms County, Norway during late autumn and winter of 2014. Lumpfish eggs were

6 collected from two wild caught females (hereafter called, batch 1 and batch 2) and put in two

7 separate plastic containers. Milt from two wild caught males was then added to both egg

8 batches. One $\mathrm{ml}$ of eggs was subtracted from each batch using a syringe and placed on two

9 petri-dishes and the eggs counted to estimate the number of eggs $\mathrm{ml}^{-1}$. Two $\mathrm{ml}$ of eggs were

10 then distributed in each experimental incubator (2 1), after having been carefully separated and

11 seawater slowly added. The experimental system used was on flow through water. Each of the

12 incubators in the experimental apparatus was made using 21 plastic bottles turned upside down

13 and stuck in a styrofoam plate. The bottom of the bottles was removed and a hole drilled in

14 the bottle-cap. A plastic plate with $1.5 \mathrm{~mm}$ mesh holes made up the bottom of the incubator.

15 The incubators were set up in three rows of 10 , with each row representing a different

16 temperature exposure groups. Two batches of eggs were incubated at three temperature

17 regimes, in five replicates (30 incubators in total, ca. 200 eggs in each):

18 1. Ambient seawater temperature $4-6^{\circ} \mathrm{C}(\mathrm{Cold}, \mathrm{C})$, average $4.7^{\circ} \mathrm{C}$;

19 2. Ambient seawater temperature for the first 10 days followed by gradual increase of

$20 \sim 1.3^{\circ} \mathrm{C} /$ day over 4 days to reach $10^{\circ} \mathrm{C}$ (Gradient, $\left.\mathrm{G}\right) ;$ and

$21 \quad 3$. Constant $10^{\circ} \mathrm{C}$ seawater (Warm, W).

22 Eggs from two replicates from each temperature regime and batch were sampled during the

23 incubation period, while three replicates from each regime and batch were left undisturbed

24 until hatching. From the unsampled incubators, 50 larvae were kept alive and fed with 0.1-0.2 
$1 \mathrm{~mm}$ pellets (AgloNorse Extra, Troms $\varnothing$ Fiskeindustri AS, Troms $\emptyset$, Norway) for 2 weeks after

2 hatching to study possible late effects on larval quality. The larvae were expected to hatch

3 around 280 day-degrees (dd); at 260 day-degrees a cap with a $0.5 \mathrm{~mm}$ mesh was put on the

4 water outlet of the incubators to avoid larval escape. The hatched larvae were kept in

5 containers similar to the incubators, but with switched water inlet and outlet. The water

6 temperature in the larval containers was $10^{\circ} \mathrm{C}$. Oxygen saturation was stable both during

7 incubation and after hatching. During incubation, the average oxygen saturation was $109.2 \%$

8 in the warm groups $108.3 \%$ in the gradient groups and $103.1 \%$ in the cold groups. After

9 hatching, it was $108.8 \%, 110.1 \%$ and $105.5 \%$ in the respective groups. Salinity was stable at

$10 \quad 33.5$ ppt during the experimental period.

11 Temperature and oxygen saturation levels were recorded daily using an OxyGuard

12 Handy Alpha (Sterner Aquatech, Ski, Norway). The temperature was measured in one

13 incubator from every temperature treatment. Originally, the water flow in each incubator was

14 set to approximately $21 \mathrm{~min}^{-1}$. Light was on during working hours, from 08:00 $\mathrm{h}$ to 16:00 $\mathrm{h}$

15 every day. Cleaning of the incubators was done if the accumulation of debris became visible.

16 The eggs were removed using a plastic spoon and a plastic pipette and cautiously tranfered to

17 a bucket with seawater at the respective temperature regime.

18 The experiment was carried out at Kraknes Research Station (Troms $\varnothing$, Norway) between

1911 March and 30 May 2015. Larval measurements and histological preparations and analyses

20 were carried out in the laboratory at the Department of Arctic and Marine Biology, University

21 of Troms $\varnothing$.

22 The experiment described has been approved by the local specialist responsible for

23 laboratory animal science, under the surveillance of the Norwegian Animal Research

24 Authority (NARA) and registered by the Authority. The experiment has thus been conducted 
1 in accordance with the laws and regulations controlling experiments on live animals in

2 Norway, i.e. the Animal Protection Act of 20 December 1974, No. 73, chapter VI sections 20-

322 and the Animal Protection Ordinance concerning Biological Experiments in Animals of 15

4 January 1996.

5

$6 \quad 2.2$ Sampling of eggs

$7 \quad$ Fertilization percentage and average egg diameters were calculated under a 8 stereomicroscope (Leica WILD M10, Wetzlar and Mannheim, Germany) by taking 15 eggs

9 from each sampling-incubator. To study the development, abnormalities and mortality of the 10 eggs incubated at different temperatures, samples (minimum $\mathrm{N}=20$ from each temperature

11 group) were taken throughout the incubation period from the sample incubators. In the first

12 two days, egg samples were taken twice a day. From day three and onwards, sampling was

13 done every second or third day until hatching occurred. A minimum of five eggs were taken

14 from each sampling incubator (i.e. 10 eggs from each batch, and thus 20 from each temperature

15 regime). The egg samples were sampled using a plastic spoon, lifting the eggs to the surface

16 and then carefully separating them. The eggs were then were put into glass vials with water

17 from the incubator until they were studied under the stereomicroscope. The eggs were

18 photographed through the ocular of the stereomicroscope using a mobile phone camera

19 (iPhone 4 and iPhone 6, Apple Inc., CA., USA) and then stored on $4 \%$ buffered formaldehyde

20 in case additional examinations were needed. Number of abnormal and dead embryos were

21 estimated from each sample.

22

232.3 Sampling of larvae 
1 At hatching 30-50 larvae from each of the triplicate incubators of both batches were

2 moved to feeding containers to be kept alive for 2 weeks after the hatching peak. Larvae stuck

3 on the water outlet or swimming in consecutive circles were excluded. All other larvae, except

4 the 50 larvae transferred to the feeding containers, were killed with an overdose of anaesthetics

5 ( ${ }^{\mathrm{TM} 18}$ Finquel, $\left.150 \mathrm{mg}^{-1}\right)$ and stored on $4 \%$ buffered formaldehyde to be examined later.

6 The larvae were hand feed during working hours at approximately 08.00, 10.00, 13.00

7 and $15.00 \mathrm{~h}$. They were given approximately $1 \mathrm{cl}$ of pellets (AgloNorse Extra, Troms $\varnothing$

8 Fiskeindustri AS, Troms $\varnothing$, Norway) each time. Half an hour after the last feeding, the excess

9 feed accumulated on the bottom and bacterial growth was rinsed away. Two weeks after peak

10 hatching, the larvae kept in the containers were taken out using a plastic pipette, killed with

11 an overdose of anaesthetics ( ${ }^{\mathrm{TM} 18}$ Finquel, $\left.200 \mathrm{mg} \mathrm{l}^{-1}\right)$ and stored on $4 \%$ buffered formaldehyde

12 for later examination.

\subsection{Examination of larvae}

15 A subsample of 20 hatched larvae from all triplicates of both batches, as well as a 16 subsample of 20 two weeks old and fed larvae were studied under a stereomicroscope. The

17 following measurements were taken: body length, yolk-sac height, body height above anal 18 opening (Fig. 1), weight, dorsal fin development, tail bend, spine damage, deformed body and 19 mechanical damage (e.g. missing body parts, bursted yolk-sac, degradation). Using tweezers, 20 the larvae were carefully picked up and rolled on a piece of paper towel to dry of excess 21 moisture, then put on a small disc made of aluminum foil and weighed. The body weights (wet 22 weight) of the larvae were measured to the nearest $0.001 \mathrm{mg}$ using a Mettler MX5 23 microbalance (Mettler-Toledo, Columbus, OH, USA). 
Hatched and two weeks old larvae (3 larvae from each of the replicates) were fixed in

3 buffered formaldehyde (4\%), embedded in wax (Paraplast, Merck, Darmstadt, Germany) and

4 sectioned longitudinally at $5 \mu \mathrm{m}$ with a rotation microtome. Sections were stained with eosin

5 and haemotoxylin and examined and photographed under a microscope (Leica DM2000 LED,

6 Wetzlar and Mannheim, Germany). Photos were taken using a Leica DFC295 camera and

7 computer software (Leica Applicationsuite V4.7, Wetzlar and Mannheim, Germany).

\section{$9 \quad 2.6$ Statistics}

10 All statistical analyses were conducted using Statistica ${ }^{\mathrm{TM}} 12.0$ software. A Kolmogorov-

11 Smirnov test (Zar, 1984) was used to assess for normality of distributions. The homogeneity

12 of variances was tested using the Levene's F test (Zar, 1984). Egg mortality was calculated

13 from the number of dead eggs found in the samples taken during incubation. Possible

14 differences in hatching percentage and larval mortality was tested with a one-way ANOVA.

15 Possible differences in length, body height, yolk-sac height, and weight of larvae were tested

16 with a two-way nested ANOVA (Zar, 1984) where replicates were nested within treatments.

17 Significant differences revealed in ANOVA were followed by Student-Newman-Keuls (SNK)

18 post hoc test to determine differences among experimental groups. Differences in dorsal fin

19 development, tail bend, spine damage, deformities and mechanical damage were recorded as

20 present or not present (binomial) and therefore analyzed using a non-parametric Kruskal-

21 Wallis ANOVA by ranks test (Zar, 1984). For the newly hatched larvae these statistical tests

22 were done when hatching occurred in a minimum of two of the temperature regimes at the

23 same days post hatching (DPH), with the first day of hatching being 0 DPH. A significance

24 level $(\alpha)$ of 0.05 was used if not stated otherwise. 


\section{3. Results}

\section{$3 \quad 3.1$ Egg size and numbers}

$4 \quad$ Egg size did not vary between the two egg batches from the two females (one way

5 ANOVA, $P>0.15)$. The mean $( \pm \mathrm{SE})$ egg diameter was $2.3 \pm 0.01 \mathrm{~mm}$ in batch 1 and $2.28 \pm 0.01$

$6 \mathrm{~mm}$ in batch 2. The number of eggs per $\mathrm{ml}$ was higher in batch 1 (109) than in batch 2 (94).

7 Thus, an estimate of 218 eggs from batch 1 and 188 from batch 2 were distributed into each 8 incubator.

$10 \quad 3.2$ Fertilization and development

11 Fertilization percentage was high in both batches; $97.8 \%$ in batch 1 and $98.9 \%$ in

12 batch 2 . Early cell symmetry (2-16 cell stage) appeared normal in all temperature regimes and

13 in both batches (Fig. 2A-E). Development from fertilization to hatching (Fig. 2) was faster

14 with increasing temperature, but in relation to day-degrees it was fairly similar between all

15 groups. Egg mortality was lowest (two-way nested ANOVA, $P<0.05$ ) in the warm group

$16(4.7 \%)$ and highest in the cold group (12.4\%) with the gradient group in between (8.8\%).

\subsection{Hatching}

Hatching started at 279 dd (28 days post fertilization (DPF)) in the warm regime,

20 reached a hatching peak (50\% of total hatching) at 3 days post hatch (DPH), and ended at 9

21 DPH. In the gradient regime hatching started at $280 \mathrm{dd}$ (35 DPF) and reached the hatching

22 peak the same day with almost $80 \%$ of all larvae hatching. Hatching ended after 7 days in this

23 group. In the cold temperature group hatcing started at $285 \mathrm{dd}$ (63 DPF) and the hatching peak

24 was reached at $3 \mathrm{DPH}$. Hatching lasted until $13 \mathrm{DPH}$. The average hatching percent (mean \pm 
1 SD) varied between the three temperature groups (SNK post hoc test, $P<0.05)$ and was

2 highest in the gradient group $(74.9 \% \pm 4.2)$, in between in the warm group $(58.3 \% \pm 2.9)$ and

3 lowest in the cold group $(46.1 \% \pm 7.2)$. Further, there was a significant difference (two-way

4 nested ANOVA, $P<0.05$ ) in mortality (mean $\pm \mathrm{SD}$ ) at hatching between the temperature

5 groups with the highest mortality $(38.5 \% \pm 15.7)$ seen in the warm group and lowest in the

6 gradient group $(13.2 \% \pm 6.3)$. Mortaliy at hatching in the cold group was in between $(27.0 \%$ $7 \quad \pm 6.8)$

\subsection{Larval development and deformities}

10 At hatch, larvae from the cold temperature regime were longer and heavier compared 11 to the other two experimental groups (SNK post hoc test, $P<0.05$, Table 1 ). The gradient 12 regime larvae had the largest yolk sacs, while the cold group larvae had the smallest (Table

13 1). The development of the dorsal fin was only noted among the warm and gradient group

14 larvae (Table 2). Larvae from the warm regime had significantly higher occurrence of bended 15 tail, spine damage, deformities and other body damages compared to the gradient and cold 16 incubation regimes (Kruskal Wallis non-parametric test, $P<0.01$, Table 2).

17 The newly hatched larvae were all reared at $10^{\circ} \mathrm{C}$ for 2 weeks. The two week old larvae 18 from the cold incubation group was longer and heavier compared to the two other experimental 19 groups (SNK post hoc test, $P<0.05$, Table 3).

\subsection{Histology}

22 The organ- and tissue histomorphology of hatched lumpfish larvae were relatively 23 mature, even if a significant yolk rest was still present (Fig. 3). The eyes were heavily

24 pigmented and appear functional, mouth and total digestive system well developed with folded 
1 and differentiated mucosa. For the two-week-old larva (Fig. 4), the liver was large with

2 vacuolated hepatic cells, pancreatic tissue and kidneys present, gill development has been

3 initiated, and the ventral suction-disc was well developed. No obvious histological differences

4 between larvae from the various temperature regimes were registered at each timepoint, 5 among neither the newly hatched larvae nor the two weeks old ones. In the two week old 6 larvae for all temperature groups, there was little or no yolk left (Fig. 4), and the 7 histomorphology did not deviate much from the newly hatched larvae. Food particles were 8 noted in the intestines of larvae from all temperature groups. 


\section{4. Discussion}

Eggs in the warm incubation group reached hatching fastest; at 28 DPF (279 dd); and

4 the cold group was the slowest at $63 \mathrm{DPF}$ (285 dd). This was expected as it is well known that

5 egg incubation time is longer at lower temperature (Mueller et al., 2015; Hu et al., 2017). The

6 slow cell division during the first cleavage stages of lumpfish eggs have been noted by others

7 as well (Davenport, 1983). However, the overall developmental rate of the eggs was very

8 similar in all temperature groups when calculated as number of day degrees. The gradual

9 increase of incubation temperature in the gradient group took place after 10 days when the

10 eggs had reached the embryo phase; i.e. after the most temperature sensitive developmental

11 phases (Kazuyuki et al., 1988).

12 There were significant differences in egg mortality between the temperature regimes,

13 with the warm group having the lowest (4.7\%) and the cold group the highest (12.4\%) total

14 mortality. In contrast Geffen et al. (2006) found that the mortality rate of Irish Sea cod eggs

15 tended to increase with incubation temperature but. Therefore, it was surprising to find in the

16 present study that the egg mortality was relatively high in the cold group, as ambient water

17 was used and the experiment took place in a period when the lumpfish spawn naturally

18 (Davenport, 1985; Moen and Svensen, 2004; Durif, 2015). One possible explanation for the

19 high egg mortality in the cold group could be that the water was excessively cold for a short

20 period of time, lowest recorded temperature was $3.8^{\circ} \mathrm{C}$. Collins (1978) reported that lumpfish

21 eggs incubated at an average temperature of $3.8^{\circ} \mathrm{C}$ degrees failed to hatch. The lumpfish lays

22 its eggs in shallow water (Davenport, 1985; Moen and Svensen, 2004, Durif, 2015), where

23 temperature stratification can take place if conditions are right. Another possibility is that

24 incubation time may be a crucial factor for survivability. The longer the incubation time is the 
1 greater the chance for infection or attack by fungi and bacteria. However, in this experiment

2 it was registered that the cold group had less problems with fouling compared to the groups

3 running on warm water.

4 Although the time of hatching varied much in days' post fertilization among the three

5 temperature regimes, 28, 35 and 63 DPF in the warm, gradient and cold group respectively,

6 hatching started at approximately 280 day degree in all groups. There was, however, a large

7 variation in how synchronized the eggs hatched. The warm and cold groups both had a slow

8 start, and reached $50 \%$ hatching at $3 \mathrm{DPH}$. The gradient groups differed from the other groups

9 as most of the eggs hatched during the first day of hatching. It is possible that the change in

10 incubation temperature the gradient group was exposed to influenced the synchronization of

11 egg hatching. Such temperature sensitive effect on synchronization of hatching has been

12 indicated for fish (Villamizar et al., 2013). Their results revealed that hatching rhythms in fish

13 are endogenously driven by a time-keeping mechanism, so that the day and time of hatching

14 are determined by the interplay between the developmental state (temperature-sensitive) and

15 the circadian clock (temperature-compensated).

16 There were significant differences in larval mortality at hatching between the

17 temperature regimes; the highest mortality was registered in the warm and the lowest in the

18 gradient groups. Mueller et al. (2015) also found increased mortality with increasing

19 incubation temperature among lake whitefish (Coregonus clupeaformis) larvae and so did

20 Geffen et al. (2006) in experiments with Atlantic cod (Gadus morhua) eggs and larvae. The

21 larval mortality among two weeks old fed larvae was not significantly different between

22 temperature groups or batches in our experiment.

23 Body measurements of the lumpfish larvae showed variations in length, weight, body

24 height and yolk-sac size. The overall impression of these data is that newly hatched larvae 
1 from the cold temperature regime were longest, heaviest and thickest, while those from the

2 warm regime were shortest, lightest and thinnest. Smaller larvae with larger yolk sacs hatching

3 from eggs incubated at higher temperature has been found in experiments with other marine

4 and freshwater fishes (Hansen and Falk-Petersen, 2001; Sund and Falk-Petersen, 2005;

$5 \quad$ Mueller et al., 2015)

6 The only gross anatomical changes observed between larvae from different incubation

7 regimes, was that the separation of the dorsal fin from the larval-finfold was only registered

8 among newly hatched larvae from the warm and gradient groups. There was some variation

9 in tail shape, spine damage, deformities and body damage within the batches and replicates,

10 however, larvae from the warm regime had overall significantly higher occurrence of such

11 abnormalities. Prevalence of malformed larvae with severe vertebral curvature has been found

12 to significantly increase with egg incubation temperature in Atlantic cod (Fitzimmons and

13 Perutz, 2006). This suggests that incubation temperature has an important influence on the

14 quality of the lumpfish larvae, and that the low temperature of $4-5^{\circ} \mathrm{C}$ early in the incubation

15 period, compared to a high of $10^{\circ} \mathrm{C}$, is more beneficial to the final quality of the larvae. The

16 higher presence of malformed larvae at hatch in the warm group correlates with the higher

17 mortality at hatch in this group.

18 No organ or tissue defects or obvious developmental differences were revealed

19 between larvae from the three incubation temperature regimes as evaluated from the

20 histological slides prepared from newly hatched larvae or two weeks old larvae. Lumpfish

21 larvae are relatively well developed at hatch (Davenport, 1985; Timeyko, 1986). From newly

22 hatched to two weeks old larvae a slightly more expanded and folded intestine was observed.

24 5. Conclusion 
1 The incubation temperature plays an important role with regard to egg mortality and

2 general quality of the lumpfish eggs and larvae. Low ambient seawater temperature $\left(4-5^{\circ} \mathrm{C}\right)$

3 during the early incubation period followed by a gradual increase to $10{ }^{\circ} \mathrm{C}$ after 10 days when

4 the early embryonic stage was reached, resulted in low egg mortality, high hatching success,

5 synchronized hatching with low mortality, medium sized larvae and few abnormal body

6 features. For producers of lumpfish larvae and juveniles this regime may represent a good

7 production regime to achieve maximum production from their egg material. At Kraknes

8 Research Station, we have used temperatures from 8 to $10^{\circ} \mathrm{C}$ with around $50 \%$ larval survival

9 from hatch to around $20 \mathrm{~g}$ weight (Thor Arne Hangstad, Kraknes Research Station, pers.

10 comm.). Future experiments should focus on temperature sensitive early stages and when and

11 how fast an increase in incubation temperature should be implemented.

\section{Acknowledgements}

14 The authors would like to thank the technical staff at Akvaplan-niva's Research and Innovation

15 Sentre at Kraknes for valuable assistance prior to and during the experimental period. Financial

16 support was given by the Norwegian Research Council (AVLUS, 239135) and by the The

17 Norwegian Seafood Research Fund (grant 900977). 


\section{References}

3 Brown, J.A., Somerton, D.C., Methven, D.A., 1992. Recent advances in lumpfish Cyclopterus

4 lumpus and ocean pout Macrozoarces americanus larviculture. Journal of the World $5 \quad$ Aquaculture Society 23, 271-276.

6 Collins, M.A.J., 1978. Experiments on the hatching period of the eggs of the lumpsfish

7 Cyclopterus lumpus L. in Newfoundland waters. Le Naturaliste Canadien 105, 169-171.

8 Davenport, J., 1983. Oxygen and the developing eggs and larvae of the lumpfish Cyclopterus

9 lumpus. Journal of Marine Biological Association United Kingdom 63, 633-640.

10 Davenport, J., 1985. Synopsis of biological data of the lumpsucker Cyclopterus lumpus (L 11 1758). FAO Fisheries synopsis No. 147.31 pp.

12 Davenport, J., Thorsteinsson, V., 1989. Observations on the colours of lumpsuckers, 13 Cyclopterus lumpus L. Journal of Fish Biology 35, 829-838.

14 Durif, C., 2015. Rognkjeks/-kall. Havforskningsraporten, Fisken og havet, særnummer 1-2015.

15 Institute of Marine Research, 183 (in Norwegian with abstract in English).

16 Fitzimmons, S.D., Perutz, M., 2006. Effects of egg incubation temperature on survival, 17 prevalence and types of malformations in vertebral column of Atlantic Cod (Gadus morhua) 18 larvae. Bulletin of the European Association of Fish Pathologists 26, 80-86.

19 Geffen, A.J., Fox C.J., Nash R.D.M., 2006. Temperature-dependent development rates of cod 20 Gadus morhua eggs. Journal of Fish Biology 69, 1060-1080.

21 Hansen, T.K., Falk-Petersen, I.-B., 2001. The influence of rearing temperature on early 22 development and growth of spotted wolffish Anarhichas minor (Olafsen). Aquaculture 23 Research 32, 369-378. 
1 Hu, F., Pan, L., Gao, F., Jian, Y., Wang, X., Li, L., Zhang, S., Guo, W., 2017. Effect of

2 temperature on incubation period and hatching success of fat greenling (Hexagrammos

3 otakii Jordan \& Starks) eggs. Aquaculture Research 48, 361-365.

4 Imsland, A.K., Reynolds, P., Eliassen, G., Hangstad, T.A., Foss, A., Vikingstad, E., Elvegård,

5 T.A., 2014a. The use of lumpfish (Cyclopterus lumpus L.) to control sea lice

6 (Lepeophtheirus salmonis Krøyer) infestations in intensively farmed Atlantic salmon

7 (Salmo salar L.). Aquaculture 425-426, 18-23.

8 Imsland, A.K., Reynolds, P., Eliassen, G., Hangstad, T.A., Nytrø, A.V., Foss, A., Vikingstad,

9 E., Elvegård, T.A., 2014b. Notes on behaviour of lumpfish in sea pens with and without

10 Atlantic salmon. Journal of Ethology 32, 117-122.

11 Imsland, A.K., Reynolds, P., Eliassen, G., Hangstad, T.A., Nytrø, A.V., Foss, A., Vikingstad,

12 E., Elvegård, T.A., 2014c. Assessment of growth and sea lice infection levels in Atlantic

13 salmon stocked in small-scale cages with lumpfish. Aquaculture 433, 137-142.

14 Imsland, A.K., Reynolds, P., Eliassen, G., Hangstad, T.A., Nytrø, A.V., Foss, A., Vikingstad,

15 E., Elvegård, T.A., 2015a. Feeding preferences of lumpfish (Cyclopterus lumpus L.)

16 maintained in open net-pens with Atlantic salmon (Salmo salar L.) Aquaculture 436, 47-

1751.

18 Imsland, A.K., Reynolds, P., Eliassen, G., Hangstad, T.A., Nytrø, A.V., Foss, A., Vikingstad,

19 E., Elvegård, T.A., 2015b. Assessment of suitable substrates for lumpfish in sea pens.

20 Aquaculture International 23, 639-645.

21 Jonsson, B. Jonsson, N., 2014. Early environment influences later performance in fishes.

22 Journal of Fish Biology 85, 151-188. 
1 Kazuyuki, S., Kurokura H., Kasahara, S., 1988. Changes in low temperature tolerance of the

2 egg of certain marine fish during embryonic development. Comparative Biochemistry and

$3 \quad$ Physiology 91A, 183-187.

4 Moen, F.E., Svensen E., 2004. Marine fish \& invertebrates of Northern Europe. AquaPress,

5 Southend-On-Sea, UK, 608 pp.

6 Mueller, C.A., Eme, J., Manzon, R.G., Somers, C.M., Boreham, D.R., Wilson J.Y., 2015.

7 Emryonic critical windows: changes in incubation temperature alter survival, hatching

8 phenotype, and cost of development in lake whitefish (Coregonus clupeaformis). Journal

9 of Compartive Physiology 185B, 315-331.

10 Rombough, P.J., 1997. The effects of temperature on embryonic and larval development. In:

11 Wood, C.M, McDonald, D.G. (Eds.), Global warming: Implications for freshwater and

12 marine fish. Cambridge University Press, Cambridge, UK, pp. 177-223.

13 Sund, T., Falk-Petersen, I.B., 2005. Effects of incubation temperature on development and

14 yolk sac conversion efficiencies of spotted wolffish (Anarhichas minor Olafsen) embryos

15 until hatch. Aquaculture Research 36, 1133-1143.

16 Timeyko, V.N., 1986. The digestive systems of white sea cod, Gadus morhua marisalbi and

17 lumpfish Cyclopterus lumpus, at different stages on ontogeny. Voprosy Ikhtiologii 26, 103-

$18 \quad 112$.

19 Treasurer, J.W., 2002. A review of potential pathogens of sea lice and the application of 20 cleaner fish in biological control. Pest Managem. Sci. 58, 546-558.

21 Villamizar, N., Blanco-Vives, B., Oliveira, C., Dinis, M.T., Di Rosa, V., Negrini, P., 22 Bertolucci, C., Sanchez-Vazquez, F.J., 2013. Circadian rhythms of embryonic development 23 and hatching in fish: a comparative study of zebrafish (Diurnal), Senegalese sole 24 (Nocturnal), and Somalian cavefish (Blind). Chronobiology International 30, 889-900. 
1 Zar, J.H., 1984. Biostatistical Analysis, 2nd edition, Prentice-Hall, Inc., Englewood Cliffs, $2 \quad$ N.J., 718 pp.

3 


\section{$1 \quad$ Figure legends}

2 Fig 1. Newly hatched lumpfish larva illustrating measurements for length (1), body height (2)

3 and yolk-sac height (3).

5 Fig. 2. Photos of lumpfish development during the egg stage (from fertilization to hatching)

6 in relation to day degrees (dd). The diameter of the eggs in A-P is $2.2 \mathrm{~mm}$.

7 A) Fertilized egg ( 1 hour post fertilization (HPF), 0 dd) with perivitelline space (arrowhead)

8 visible; B) 2-cell stage (7 HPF. 0 dd); C) 4-cell stage (4.1 dd); D) 8-cell stage (4.1 dd); E) 16-

9 cell stage (4.1 dd); F) 64-cell stage (8 dd); G) Morula (10.1 dd); H) Blastula (20.1 dd); I)

10 Gastrula (29.2 dd); J) Embryo with optic vesicle (arrowhead, 49.8 dd); K) Embryo with

11 segmentation and compression of yolk lipids (70.3 dd); L) Embryo with otocysts and more

12 developed eyes (arrowhead, $89.4 \mathrm{dd}$ ); M) Eye pigmentation and otoliths (arrowhead, 117.3

$13 \mathrm{dd}$ ); N) Heartbeat, visible vein in yolk-sac (arrowhead, $128.9 \mathrm{dd}$ ) and weak body pigmentation

14 (138.4 dd); O) Capillary network on yolk-sac (arrowhead, 173.8 dd); P) Large embryo ready

15 to hatch (278.6 dd).

17 Fig. 3. Longitudinal section of a newly hatched lumpfish larva with; brain (B), eyes (E), gills $18(\mathrm{G})$, intestine (I), liver (L), notochord $(\mathrm{N})$, pancreas $(\mathrm{P})$ and yolk-sac $(\mathrm{Y})$.

20 Fig. 4. Longitudinal section of a two weeks old lumpfish larva, anus (A) brain (B), eyes (E),

21 gills $(\mathrm{G})$, intestine $(\mathrm{I})$, liver $(\mathrm{L})$, kidney $(\mathrm{K})$, notochord $(\mathrm{N})$, Otocyst $(\mathrm{O})$, pancreas $(\mathrm{P})$ and 22 ventral suction-disc (S). 


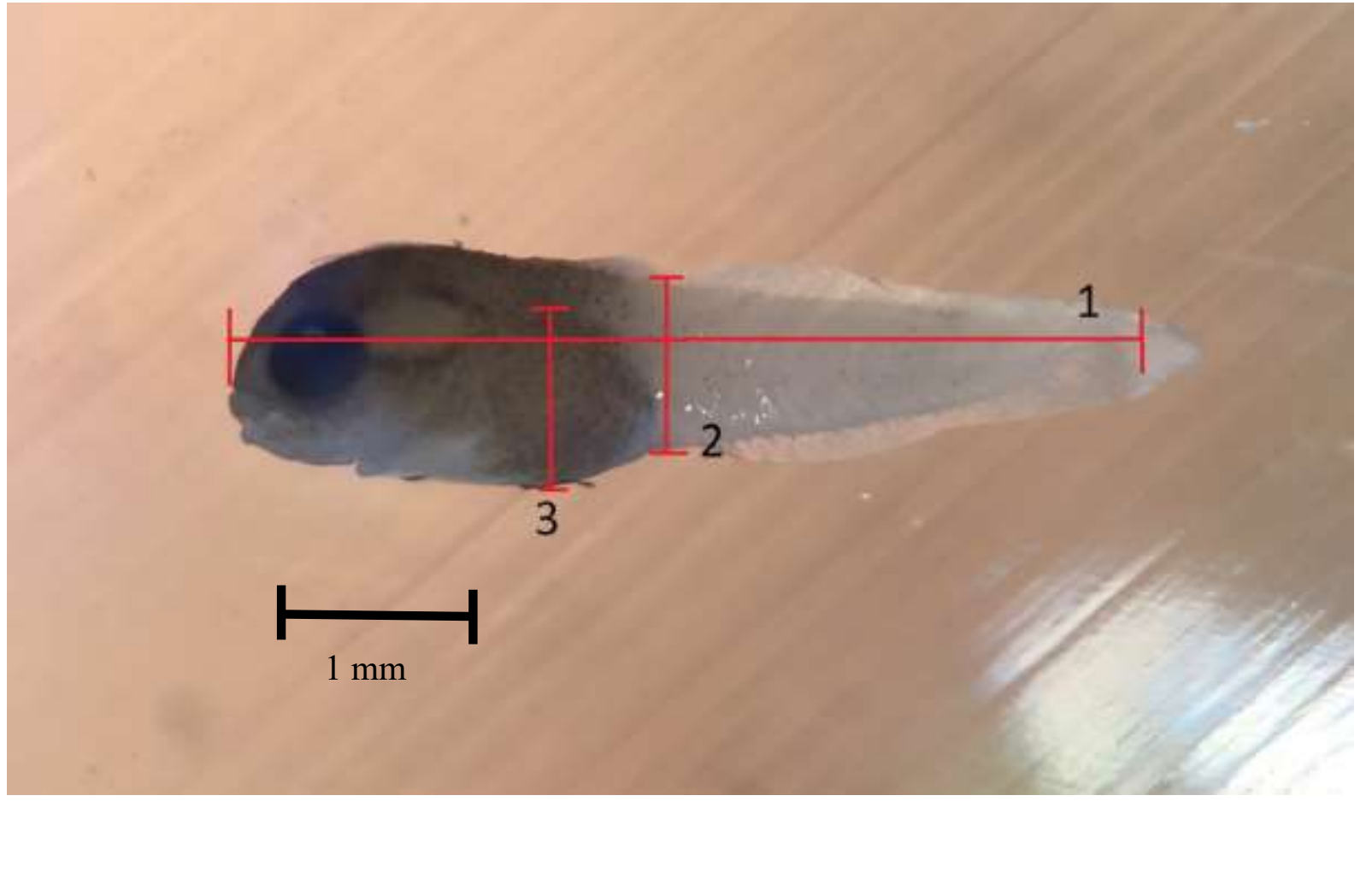

2

3 Fig. 1. Imsland et al.

4 


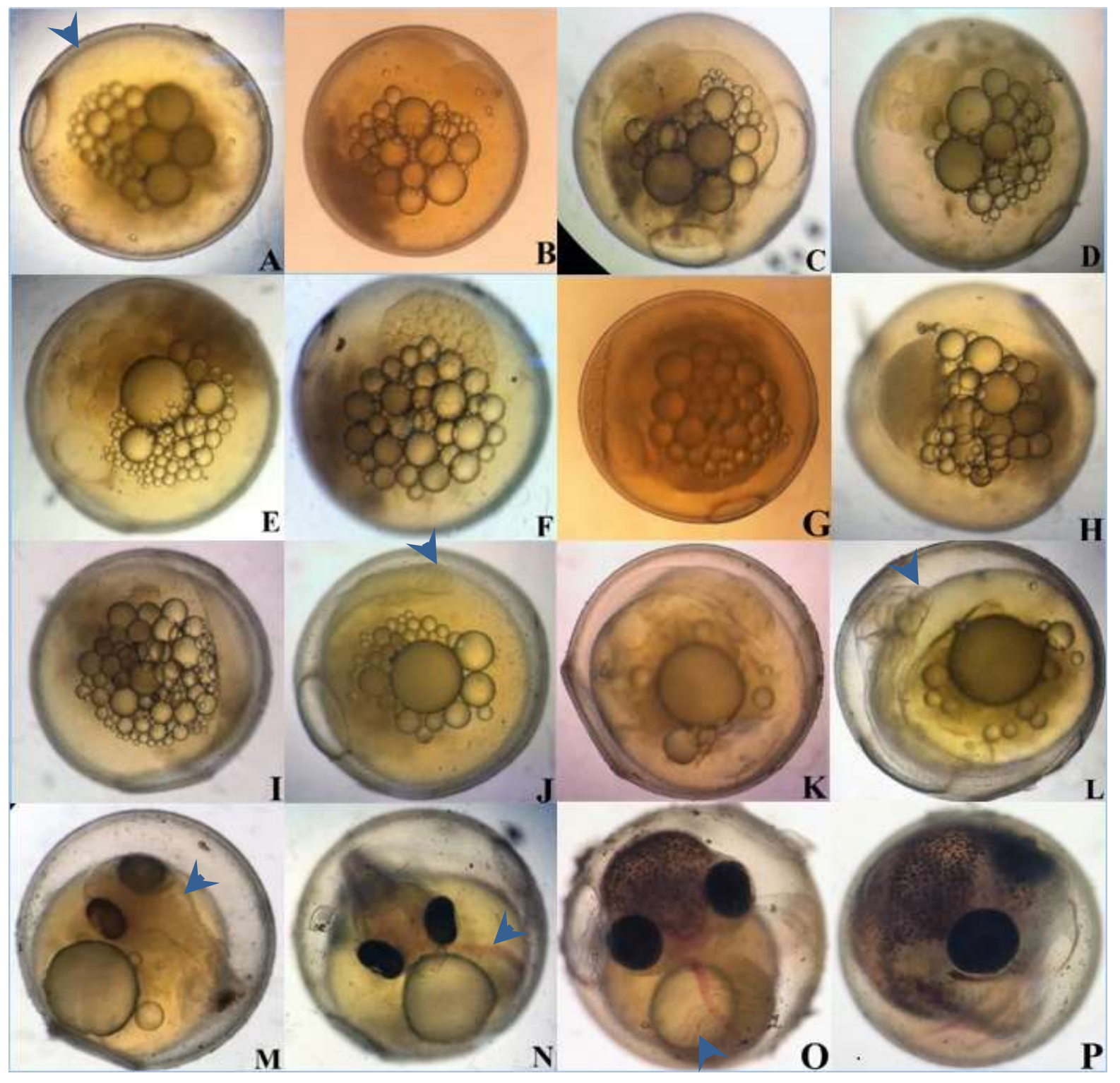

2 Fig. 2. Imsland et al. 


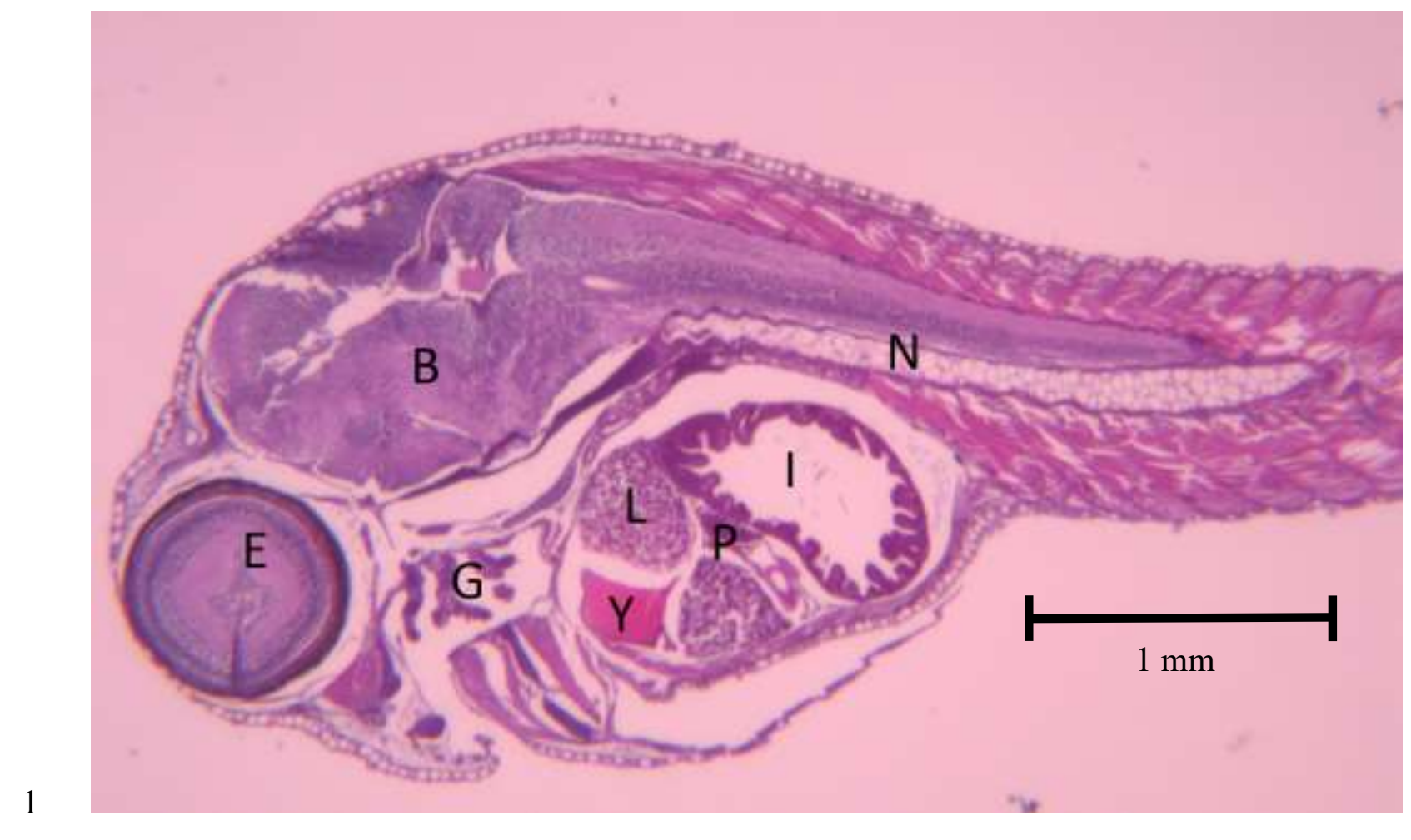

2 Figure 3. Imsland et al.

3

4 


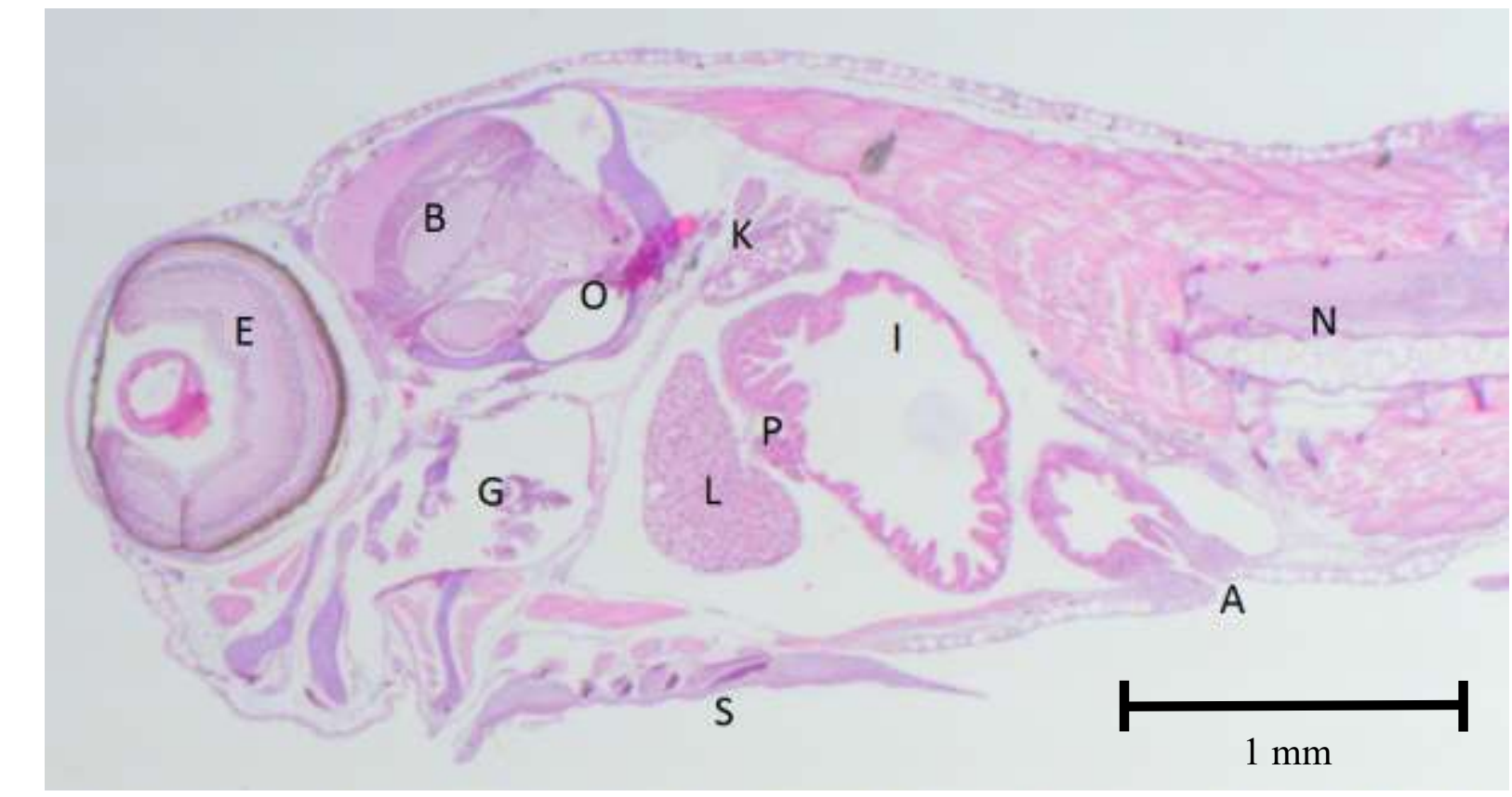

3 Fig. 4. Imsland et al.

4 
1 Table 1. Average (SD) length (mm), height (mm), yolk-sac height (mm) and weight (mg) for

2 newly hatched lumpfish larvae during 0-10 DPH. Values followed by different superscripted

3 letters are significant different (SNK post hoc test, $P<0.05$ ). $\mathrm{N}=60$ for each experimental

4 group.

\begin{tabular}{lcccc}
\hline Group & Length & Height & Yolk-sac height & Weight \\
\hline Warm & $5.33(0.85)^{\mathrm{c}}$ & $0.95(0.14)^{\mathrm{c}}$ & $1.12(0.08)^{\mathrm{b}}$ & $4.37(0.75)^{\mathrm{c}}$ \\
Gradient & $5.71(0.64)^{\mathrm{b}}$ & $1.02(0.14)^{\mathrm{b}}$ & $1.15(0.08)^{\mathrm{a}}$ & $4.88(0.79)^{\mathrm{b}}$ \\
Cold & $6.11(0.72)^{\mathrm{a}}$ & $1.08(0.14)^{\mathrm{a}}$ & $1.11(0.09)^{\mathrm{b}}$ & $5.55(0.84)^{\mathrm{a}}$ \\
& & & & \\
\hline
\end{tabular}

5

6 
1 Table 2. Average occurrence (SD) of dorsal fin, tail bend, spine damage, deformities body and

2 damaged body for newly hatched lumpfish larvae during 0-10 DPH. Values followed by

3 different superscripted letters are significant different (Kruskal Wallis non parametric test, $P$

$4<0.05) . \mathrm{N}=60$ for each experimental group.

\begin{tabular}{llcccc}
\hline Group & Dorsal fin & Tail bend & Spine damage & Deformed & Damaged \\
\hline Warm & $20.1(22.1)^{\mathrm{a}}$ & $44.9(15.9)^{\mathrm{a}}$ & $35.0(16.0)^{\mathrm{a}}$ & $48.13(37.9)^{\mathrm{a}}$ & $25.2(23.1)^{\mathrm{a}}$ \\
Gradient & $8.63(8.2)^{\mathrm{a}}$ & $12.2(0.1)^{\mathrm{b}}$ & $3.1(2.7)^{\mathrm{b}}$ & $18.4(9.9)^{\mathrm{b}}$ & $2.3(1.7)^{\mathrm{b}}$ \\
Cold & $<0.01(<0.1)^{\mathrm{b}}$ & $11.1(8.4)^{\mathrm{b}}$ & $8.0(5.6)^{\mathrm{b}}$ & $15.6(10.6)^{\mathrm{b}}$ & $4.0(4.2)^{\mathrm{b}}$ \\
& & & & & \\
\hline
\end{tabular}

5

6 
1 Table 3. Average (SD) length ( $\mathrm{mm})$, height $(\mathrm{mm})$ and weight $(\mathrm{mg})$ for two week old lumpfish

2 larvae incubated at different temperatures. Values followed by different superscripted letters

3 are significant different ( $\mathrm{SNK}$ post hoc test, $P<0.05$ ). $\mathrm{N}=60$ for each experimental group.

\begin{tabular}{llll}
\hline Group & Length & Height & Weight \\
\hline Warm & $6.44(0.61)^{\mathrm{b}}$ & $1.16(0.08)^{\mathrm{a}}$ & $7.07(0.56)^{\mathrm{b}}$ \\
Gradient & $6.42(0.23)^{\mathrm{b}}$ & $1.15(0.08)^{\mathrm{a}}$ & $6.71(0.62)^{\mathrm{c}}$ \\
Cold & $6.67(0.24)^{\mathrm{a}}$ & $1.12(0.08)^{\mathrm{b}}$ & $7.82(0.86)^{\mathrm{a}}$ \\
\hline
\end{tabular}

4

5 